\title{
Article \\ Proximal Tibiofibular Dislocation in Closing-Wedge High Tibial Osteotomy Increases the Risk of Medium and Long-Term Total Knee Replacement
}

\author{
Juan Sánchez-Soler ${ }^{1,2, *}$, Alex Coelho ${ }^{1}$ (D), Raúl Torres-Claramunt 1,2,3, Berta Gasol ${ }^{1}$, Albert Fontanellas ${ }^{1}$, \\ Simone Perelli ${ }^{1,3}$, Pedro Hinarejos ${ }^{1,2}$ and Joan Carles Monllau 1,2,3 \\ 1 Department of Surgery and Morphologic Science, Orthopaedic Surgery Service, Universitat Autònoma \\ de Barcelona, Hospital del Mar, 08003 Barcelona, Spain; 63611@parcdesalutmar.cat (A.C.); \\ rtorresclaramunt@psmar.cat (R.T.-C.); bgasolcudos@gmail.com (B.G.); 64292@parcdesalutmar.cat (A.F.); \\ sperelli@parcdesalutmar.cat (S.P.); phinarejos@hospitaldelmar.cat (P.H.); \\ jmonllau@parcdesalutmar.cat (J.C.M.) \\ 2 IMIM (Hospital del Mar Medical Research Institute), 08003 Barcelona, Spain \\ 3 Catalan Institute for Traumatology and Sports Medicine (ICATME), Hospital Universitari Dexeus, UAB, \\ 08028 Barcelona, Spain \\ * Correspondence: jfsanchez@parcdesalutmar.cat
}

Citation: Sánchez-Soler, J.; Coelho, A.; Torres-Claramunt, R.; Gasol, B.; Fontanellas, A.; Perelli, S.; Hinarejos, P.; Monllau, J.C. Proximal Tibiofibular Dislocation in Closing-Wedge High Tibial Osteotomy Increases the Risk of Medium and Long-Term Total Knee Replacement. J. Clin. Med. 2021, 10, 2743. https://doi.org/10.3390/ jcm10132743

Academic Editor:

Vicente Sanchis-Alfonso

Received: 14 May 2021

Accepted: 18 June 2021

Published: 22 June 2021

Publisher's Note: MDPI stays neutral with regard to jurisdictional claims in published maps and institutional affiliations.

Copyright: (c) 2021 by the authors. Licensee MDPI, Basel, Switzerland. This article is an open access article distributed under the terms and conditions of the Creative Commons Attribution (CC BY) license (https:/ / creativecommons.org/licenses/by/ $4.0 /)$.
Abstract: Proximal tibiofibular dislocation in closing-wedge high tibial osteotomy increases the risk of medium and long-term total knee replacement. Background: High tibial osteotomy is an effective treatment for medial osteoarthritis in young patients with varus knee. The lateral closing-wedge high tibial osteotomy (CWHTO) may be managed with tibiofibular dislocation (TFJD) or a fibular head osteotomy (FHO). TFJD may lead to lateral knee instability and thereby affect mid- and long-term outcomes. It also brings the osteotomy survival rate down. Objective: To compare the CWHTO survival rate in function of tibiofibular joint management with TFJD or FHO, and to determine whether medium and long-term clinical outcomes are different between the two procedures. Material $\mathcal{E}$ Methods: A retrospective cohort study was carried out that included CWHTO performed between January 2005 to December 2018. Those patients were placed in either group 1 (FHO) or Group 2 (TFJD). Full-leg weight-bearing radiographs were studied preoperatively, one year after surgery and at final follow-up to assess the femorotibial angle (FTA). The Rosenberg view was used to assess the Ahlbäck grade. The Knee Society Score (KSS) was used to assess clinical outcomes and a Likert scale for patient satisfaction. The total knee replacement (TKR) was considered the end of the follow-up and the point was to analyze the CWHTO survival rate. A sub-analysis of both cohorts was performed in patients who had not been FTA overcorrected after surgery (postoperative FTA $\leq 180^{\circ}$, continuous loading in varus). Results: A total of 230 knees were analyzed. The follow-up period ranged from 24-180 months. Group 1 (FHO) consisted of 105 knees and group 2 (TFJD) had 125. No preoperative differences were observed in terms of age, gender, the KSS, FTA or the Ahlbäck scale; neither were there any differences relative to postop complications. The final follow-up FTA was $178.7^{\circ}$ (SD 4.9) in group 1 and $179.5^{\circ}$ (SD 4.2) in group 2 ( $p=0.11$ ). The Ahlbäck was 2.21 (SD 0.5) in group 1 and 2.55 (SD 0.5) in group $2(p=0.02)$ at the final follow-up. The final KSS knee values were similar for group $1(86.5 \pm 15.9)$ and group $2(84.3 \pm 15.8)$. Although a non-significant trend of decreased HTO survival in the TFJD group was found $(p=0.06)$ in the sub-analysis of non-overcorrected knees, which consisted of 52 patients from group 1 (FHO) and 58 from group 2 (TFJD), $12.8 \%$ of the patients required TKR with a mean of 88.8 months in group 1 compared to $26.8 \%$ with a mean of 54.9 months in the case of group $2(p=0.005)$. However, there were no differences in clinical and radiological outcomes. Conclusion: TFJD associated with CWHTO shows an increase in the conversion to TKR at medium and long-term follow-up with lower osteotomy survival than the CWHTO associated with FHO, especially in patients with a postoperative FTA $\leq 180^{\circ}$ (non-overcorrected). There were no differences in clinical, radiological or satisfaction results in patients who did not require TKR. Level of evidence III. Retrospective cohort study. 
Keywords: closing-wedge osteotomy; knee stability; tibiofibular dislocation; fibular osteotomy; high tibial osteotomy; osteotomy survival rate; total knee replacement; knee

\section{Introduction}

High tibial osteotomy (HTO) is a joint-preserving procedure that is widely accepted as an effective treatment for young patients with isolated medial compartment osteoarthritis $(\mathrm{OA})$ in varus knee [1,2]. The purpose of the procedure is to transfer weight-bearing forces from the medial to the lateral knee compartment to reduce the load and contact area over the previously affected compartment. The most commonly used techniques include the lateral closing-wedge HTO (CWHTO) and the medial open-wedge HTO (OWHTO) [3]. Over recent years, OWHTO has gained popularity for the treatment of symptomatic varus knees. However, there is some controversy as to whether there are functional and radiological differences between the two procedures [4,5].

Although OWHTO has been associated with higher non-union rates and donor site morbidity, one of the main reported disadvantages of CWHTO is tibiofibular joint (TFJ) manipulation with either TFJ dislocation (TFJD) or fibular osteotomy [6,7]. One of the major perioperative complications after fibular head osteotomy (FHO) is peroneal nerve disfunction. The reported incidence of symptomatic injury stands at between 3-20\% [8]. On the other hand, since lateral collateral ligament (LCL) and popliteus-fibular ligament (PFL) originate on the fibular head, TFJD may lead to fibular head rise and a shift in ligament tensioning with a potential impact on lateral knee laxity [9].

Scarce data has been published about lateral knee stability that compares the two procedures used for FTJ manipulation. Torres-Claramunt et al. reported that lateral knee compartment gapping is greater when a TFJD is performed instead of a fibular head osteotomy (FHO) at 1 year after an HTO without affecting clinical results [9].

Different factors have been described that lower the CWHTO survival rate. They include preoperative osteoarthritis grade $>2$ (Ahlbäck), female gender, obesity and being over 50 years old. However, there is no data analyzing whether lateral knee stability has an effect on progression to knee arthroplasty [10-12]. Since TFJD leads to greater lateral gapping, a dynamic varus knee may ensue, thus leading to worse mid- and long-term outcomes and lower CWHTO survival rates. This is especially so if no overcorrection is achieved after surgery.

The aim of this study was to determine whether two different ways of surgically manipulating the TFJ affect HTO survival rates and if this has an influence on the clinical medium and long-term results in those patients who have undergone a CWHTO. The main hypothesis of this study is that a TFJD increases lateral compartment gapping as it leads to joint instability, worsening CWHTO outcomes and could possibly reduce the survivorship of this procedure.

\section{Methods}

It is a retrospective cohort study that included all lateral CWHTO performed by seven expert knee surgeons in the same center from January 2005 to December 2018. For the study, patients were divided into two cohorts depending on the technique used over the proximal TFJ: group 1 in the case of FHO and group 2 in the case of TFJD. The study was approved by the ethics committee of our institution (2019/8762/I).

\subsection{Subjects}

The indication for osteotomy was medial compartment pain in a relatively young and active patient with medial varus knee osteoarthritis with or without prior medial meniscectomy, excluding patients with post-traumatic osteoarthritis or other previous surgeries that were not a simple meniscectomy.

Preoperative demographic variables like age, gender, laterality and BMI were collected. 


\subsection{Surgical Procedure}

All patients underwent a lateral CWHTO with the same surgical approach, an osteotomy at the same level under fluoroscopy control as well as fixation. It was done with the Natural-Knee ${ }^{\circledR}$ High Tibial Osteotomy (HTO) System (Zimmer ${ }^{\circledR}$, Warsaw, IN, USA) or conventional Coventry staples. It depended on the surgeon's preference. In all cases, a prior arthroscopy was performed with or without medial meniscectomy and knee compartment revision prior to osteotomy. The objective was the correction of the mechanical axis at the Fujisawa point. Depending on surgeon preference, FHO or TFJD was performed (Figure 1) prior to tibial osteotomy.

a

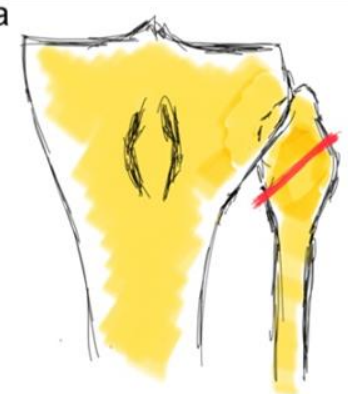

b

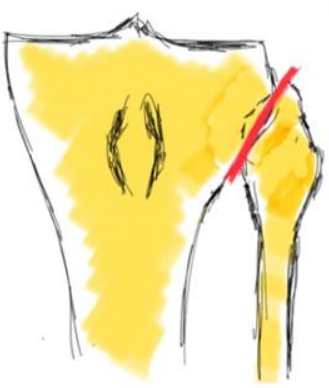

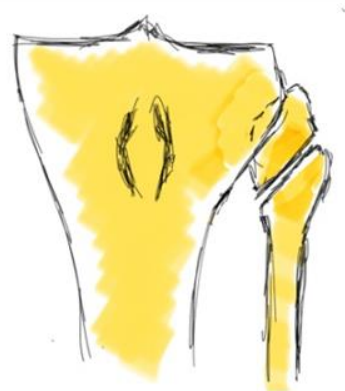

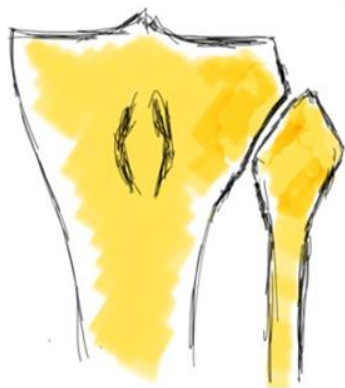

Figure 1. Fibular head osteotomy (a) and tibiofibular joint dislocation (b). Figure reproduced from [9].

\subsection{Radiological Evaluation}

A weight-bearing full-leg length $x$-ray was collected preoperatively, one year after surgery, and at the end of the follow-up for all the patients to measure the femorotibial angle (FTA) using the mechanical axis (hip-knee-ankle angle). The Rosenberg view was used to evaluate medial compartment osteoarthritis using the Ahlbäck scale. The radiological study was performed on the PACS computer system (Picture Archiving and Communication System).

\subsection{Clinical Evaluation}

The Knee Society Score (KSS) [13], specifically the Spanish version [14], was used for clinical and functional evaluation preoperatively and at the end of the follow-up (range: 24 to 180 months). The five-point Likert scale was used to measure satisfaction [15]. Moreover, patients were asked if they would undergo surgery again knowing the result obtained (yes/no) at the end of follow-up.

Surgical complications related to the procedure were collected. TKR was considered the endpoint to analyze the survival rate of CWHTO.

\subsection{Statistical Analysis}

For the initial statistical analysis, quantitative variables were described with mean and standard deviation. Qualitative variables were described with frequency tables (number and percentage). Between-group comparisons were tested with the Mann-Whitney U test. Kaplan-Meier survival curves were performed for TKR events relative to the proximal FTJ. The log-rank test was performed to check for differences between survival curves. 
Additionally, between-curve differences were checked at several time points throughout the follow-up. STATA version 15.1 (StataCorp, College Station, TX, USA) was used for statistical analysis. $p$-values $\leq 0.05$ were considered statistically significant.

A sub-analysis of both cohorts was performed on patients who had not been FTA overcorrected after surgery (postoperative FTA $\leq 180^{\circ}$, continuous loading in varus).

\section{Results}

A total of 290 knees of 272 patients were operated on, 6 died, and 48 were lost to follow-up. Finally, 230 knees of 216 patients were analyzed and follow-up ranged from 24-180 months.

There were 105 FHO knees and 125 proximal TFJD knees. Table 1 gives a demographic data summary and the preoperative radiological evaluation of both groups. No preop differences were found.

Table 1. Demographic data, preoperative KSS knee and function, femorotibial angle (FTA) and Ahlbäck scale.

\begin{tabular}{cccc}
\hline Title & Group 1. FHO $(\boldsymbol{n}$ 105) & Group 2. TFJD ( $\boldsymbol{n ~ 1 2 5 )}$ & $p$-Value \\
\hline Age (years) & $53.2(\mathrm{SD} 9.2)$ & $54.9(\mathrm{SD} 8.4)$ & n.s. \\
Biological sex (male $/$ female) & $70 / 35$ & $81 / 44$ & n.s. \\
Body mass index $\left(\mathrm{Kg} / \mathrm{m}^{2}\right)$ & $30.2(\mathrm{SD} 7.4)$ & $29.2(\mathrm{SD} 4.6)$ & n.s. \\
Side (right/left) & $47 / 58$ & $64 / 61$ & n.s. \\
Previous FTA & $170.9^{\circ}(\mathrm{SD} \mathrm{3.6})$ & $171.6^{\circ}(\mathrm{SD} 2.3)$ & n.s. \\
Ahlbäck preop & $1.9(\mathrm{SD} 0.3)$ & $2.1(\mathrm{SD} 0.4)$ & n.s. \\
KSS knee & 53.9 & 54.7 & n.s. \\
KSS function & 69.8 & 71.3 & n.s. \\
\hline
\end{tabular}

No differences were found in terms of complications, reoperation for infection or acute osteotomy failure, considered loss of correction in the first 3 months (Table 2).

Table 2. Infection and acute osteotomy fail.

\begin{tabular}{cccc}
\hline Title & Group 1. FHO $(\boldsymbol{n}$ 105) & Group 2. TFJD $(\boldsymbol{n}$ 125) & $p$-Value \\
\hline Infection & 7 & 9 & n.s. \\
Osteotomy failure & 3 & 4 & n.s. \\
\hline
\end{tabular}

Table 3 shows comparative clinical and radiological results. No differences were seen in the KSS. Additionally, there was no statistically significant difference in the conversion to TKR at the end of follow-up. In general, the patients were satisfied with the outcome of the procedure and the majority of them would be operated on again independently of the technique used for the TFJ. A statistically significant difference was found in the mean follow-up time, which was longer in the case of FHO. There was also a statistically significant difference on the Ahlbäck scale at the end of the follow-up with a greater progression in the case of TFJ dislocation.

A non-significant trend of less survival in the TFJD group was found (Figure 2) even though there was a tendency towards a significant difference at a longer follow-up time (Table 4). 
Table 3. Results summary in function of the FHO or TFJD group: clinical results (KSS R and KSS R), radiological 1 year follow-up (FTA 1y FU and Ahlbäck 1y FU), radiological final follow-up (FTA final FU and Ahlbäck final FU), mean of follow-up, FTA undercorrected, number of TKR (TKR final FU), time until TKR since HTO in months (Time to TKR), Likert scale of satisfaction (1-5) and if the patient would be operated on again (Would you repeat surgery?, yes/no).

\begin{tabular}{|c|c|c|c|}
\hline Title & Group 1. FHO ( $n$ 105) & Group 2. TFJD ( $n$ 125) & $p$-Value \\
\hline Mean Follow-Up (FU) & 109.8 months (SD 45.6) & 87.3 months (SD 32.3) & 0.01 \\
\hline KSS R & $86.5(\mathrm{SD} 15.9)$ & $84.3(\mathrm{SD} 15.8)$ & 0.33 \\
\hline KSS F & 79.7 (SD 23.2) & $77.6(\mathrm{SD} 20.6)$ & 0.31 \\
\hline FTA 1y FU & $178.9^{\circ}(\mathrm{SD} 4.8)$ & $179.9^{\circ}(\mathrm{SD} 3.8)$ & 0.12 \\
\hline FTA final FU & $178.7^{\circ}(\mathrm{SD} 4.9)$ & $179.5^{\circ}(\mathrm{SD} 4.2)$ & 0.11 \\
\hline Undercorrected FTA $\leq 180^{\circ}$ & $52(49.5 \%)$ & $58(46.4 \%)$ & 0.13 \\
\hline Ahlbäck 1y FU & $2.07(\mathrm{SD} 0.6)$ & $2.27(\mathrm{SD} 0.4)$ & 0.06 \\
\hline Ahlbäck final FU & 2.21 (SD 0.5) & $2.55(0.5)$ & $0.02 *$ \\
\hline TKR final FU & $14(13.3 \%)$ & $21(16.8 \%)$ & 0.58 \\
\hline Time to TKR & 74.4 months (SD 45.5) & 61.36 months (SD 28.6) & 0.16 \\
\hline Satisfaction (Likert 1-5) & $3.8(\mathrm{SD} 1.1)$ & $3.8(\mathrm{SD} 1.2)$ & 0.97 \\
\hline Would you repeat surgery? (yes/no) & $90 / 15(85.7 \%)$ & $102 / 23(81.6 \%)$ & 0.55 \\
\hline
\end{tabular}

* statistically significant difference.

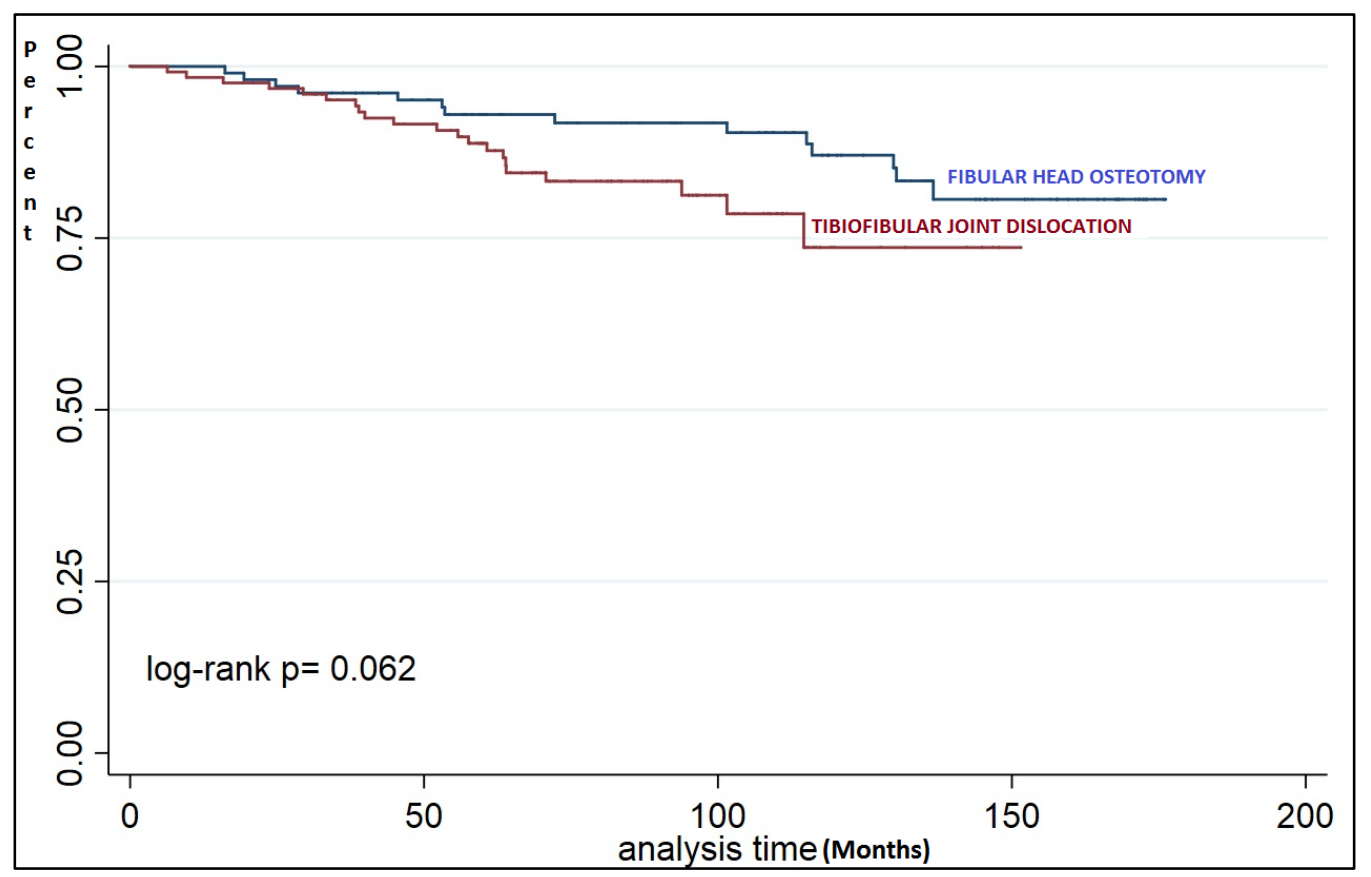

Figure 2. Survival analysis groups (log-rank test).

Table 4. Differences in survival percentages at different time points.

\begin{tabular}{cccc}
\hline Time Follow-Up (Months) & Group 1. FHO $(\boldsymbol{n}$ 105) & Group 2. TFJD $(\boldsymbol{n}$ 125) & $\boldsymbol{p}$-Value \\
\hline 24 & $98 \%$ & $96.8 \%$ & 0.53 \\
36 & $96.1 \%$ & $95.1 \%$ & 0.71 \\
48 & $95.1 \%$ & $91.6 \%$ & 0.29 \\
60 & $93 \%$ & $88.8 \%$ & 0.28 \\
120 & $87 \%$ & $73.6 \%$ & 0.07 \\
\hline
\end{tabular}

The first postoperative control radiography with an FTA angle $\leq 180^{\circ}$ (non-overcorrected) was done on 52 knees from the FHO group (49.5\%) and 58 of the TFJD group (46.4\%). In the analysis of the subgroups of non-overcorrected patients, in addition to continuing to observe the difference in the Ahlbäck scale at the end of follow-up, a significant difference was found at the TKR endpoint at the end of follow-up. A TKR was required for $12.8 \%$ of the FHO 
patients with a mean of 88.8 months from CWHTO compared to $26.8 \%$ with a mean of 54.9 months in the case of TFJD (Table 5).

Table 5. Results summary relative to the FHO or TFJD group in non-overcorrected patients, FTA $\leq 180^{\circ}$ : clinical results (KSS R and KSS R), radiological 1 year follow-up (FTA 1y FU and Ahlbäck 1y FU), radiological final follow-up (FTA final FU and Ahlbäck final FU), number of TKR (TKR final FU), time until TKR since HTO in months (Time to TKR), Likert scale of satisfaction (1-5) and if the patient would be operated again (Would you repeat surgery?, yes/no).

\begin{tabular}{cccc}
\hline Title & Group 1. FHO $(\boldsymbol{n}$ 52) & Group 2. TFJD $(\boldsymbol{n}$ 58) & $p$-Value \\
\hline KSS R & 86.9 (SD 15.7) & 83.3 (SD 17.4) & 0.4 \\
KSS F & 80.7 (SD 24.8) & 78.1 (SD 20.9) & 0.33 \\
FTA 1y FU & $176.1^{\circ}$ (SD 3.7) & $177.1^{\circ}$ (SD 2.9) & 0.24 \\
FTA final FU & $175.6^{\circ}$ (SD 3.9) & $176.5^{\circ}$ (SD 4.2) & 0.48 \\
Ahlbäck 1y FU & 2.11 (SD 0.6) & 2.25 (SD 0.5) & 0.32 \\
Ahlbäck final FU & 2.27 (SD 0.5) & $2.6(0.5)$ & $0.04^{*}$ \\
TKR final FU & $9(17.3 \%)$ & $18(31 \%)$ & $0.04^{*}$ \\
Time to TKR (months) & 88.8 (SD 45.7) & $54.9($ SD 28.3) & $0.03^{*}$ \\
Satisfaction (Likert 1-5) & 3.9 (SD 1.1) & 3.7 (SD 1.3) & 0.67 \\
Would you repeat surgery? (yes/no) & $58 / 12(82.8 \%)$ & $55 / 12(82 \%)$ & 0.59 \\
\hline *. statistically significant difference. & & &
\end{tabular}

In patients who had not required TKR, no differences were found in terms of clinical outcomes, changes in the FTA during follow-up or relative to satisfaction.

In the subgroup analysis of non-overcorrected patients, significant differences were found in osteotomy survival depending on the technique used on the proximal tibiofibular joint (Figure 3). If we analyze survival by time ranges, we find a significant difference after 60 months of follow-up (Table 6).

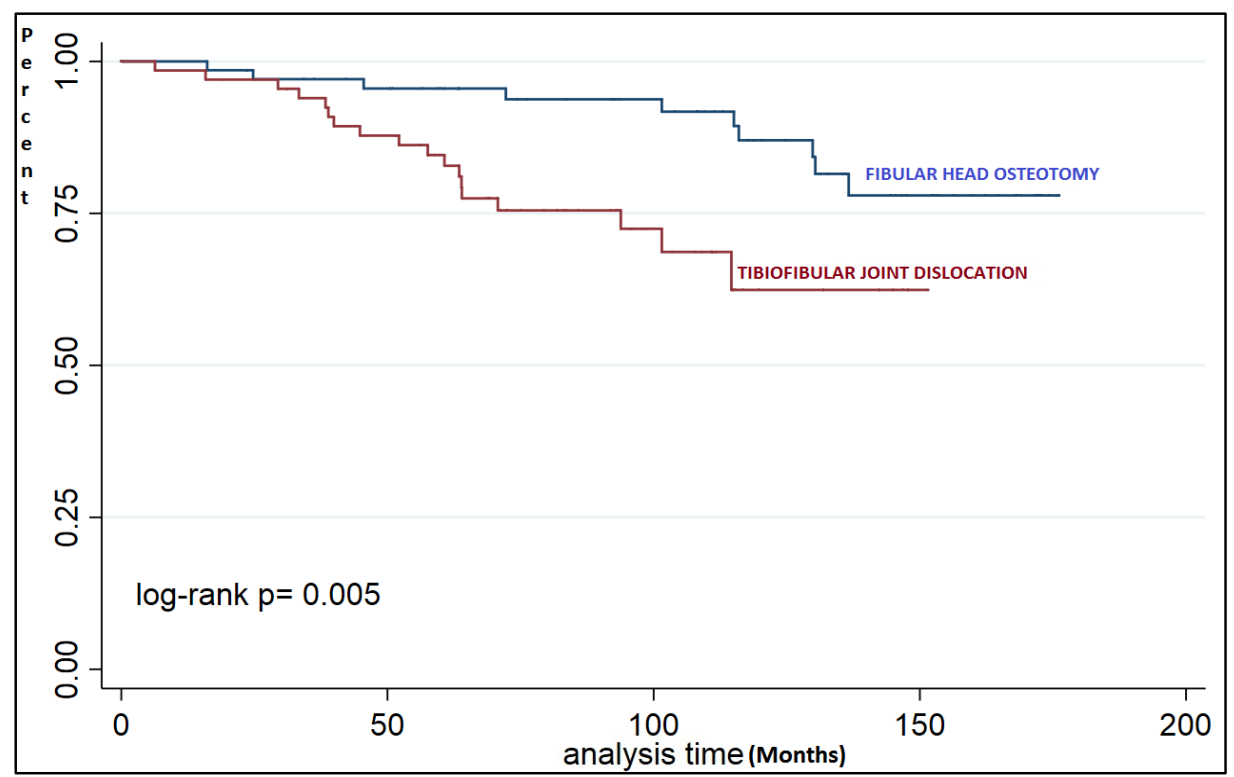

Figure 3. Survival analysis of non-overcorrected patients, FTA $\leq 180^{\circ}$ (log-rank test).

Table 6. Differences in the percentage of survival at different time points in non-overcorrected patients.

\begin{tabular}{cccc}
\hline Time Follow-Up (Months) & Group 1. FHO $(\boldsymbol{n}$ 52) & Group 2. TFJD $(\boldsymbol{n}$ 58) & $p$-Value \\
\hline 24 & 98.5 & 97 & 0.54 \\
36 & 97 & 93.9 & 0.38 \\
48 & 95.5 & 87.8 & 0.14 \\
60 & 95.5 & 84.6 & 0.03 \\
120 & 82.6 & 62.4 & 0.01 \\
\hline
\end{tabular}




\section{Discussion}

The main finding of this study is that, in the medium and long-term, patients who have undergone CWHTO in association with a TFJD progress to TKR conversion in less time when compared to patients who undergo it with an associated FHO. This finding is quite significant in those patients who are undercorrected after HTO and that present FTA is still in varus after surgery. We also found a significant progression on the Ahlbäck scale, being also worse in its progression in those patients with TFJD.

Catherine Hui et al. analyzed the survival rate of CWHTO in almost 400 patients and obtained results similar to those arrived at in our study. They observed $95 \%$ at the 5 - and $79 \%$ at 10 -year follow-up. In our series, we obtained $93 \%$ and $87 \%$ at 5 and 10 years in association with $\mathrm{FHO}$ and $89 \%$ and $73.6 \%$ in cases of TFJD.

Contrary to the initial hypothesis, we did not find relevant clinical differences in patients who completed follow-up without TKR. They presented the same values in the KSS R and KSS F independently of whether a TFJD or FHO had been done. In the medium and long-term, the progression of the FTA was similarly independent of the procedure in proximal TFJ. Therefore, we could not associate a lower survival for HTO to a progression of varus deformity.

We did not find prospective or retrospective clinical studies that evaluated different ways of treating the proximal tibiofibular joint in CWHTO to compare our results to.

Torres et al. [9] carried out a prospective randomized study performing TFJD or FHO in CWHTO, assessing lateral stability by stress radiology and functional results with KSS $\mathrm{R}$ and KSS F at 1 year follow-up. They concluded that lateral laxity increases with PDJT although without clinical differences at one year in both procedures, suggesting that it is possible clinical differences could appear in the long term due to the residual instability observed, especially in patients with postoperative varus. Continuous stress in the lateral compartment with insufficient stability could result in a greater progression of the residual varus deformity. We did not find these clinical differences, and we cannot affirm that the lower survival for HTO in the TFJD group is due to this fact, since we did not study the stability of the lateral ligament complex in our analysis, and we did not observe a different progression of the varus in any case.

A theory to explain the lower survival rate of the TFJD group is that lateral laxity may cause lower survival for HTO in some patients, an increase in arthropathy in load (change in the Ahlbäck scale) and a greater conversion to TKR in a dynamic way without objective progression of the deformity of the FTA in radiological study. Another theory is that these differences are due to the beneficial therapeutic effect of the proximal fibula osteotomy independently added to the HTO. In the last 5 years, some studies have been published, the majority biomechanical [16-20] and clinical [21-27] trials mainly from the Asian continent, which indicate that isolated FHO can reduce the pressure of the medial femorotibial compartment, improving the symptoms of varus knee osteoarthritis.

In 2015, Yang et al. [16] published the first clinical series with 156 cases of isolated proximal fibular osteotomy with 2 years of follow-up, and they conclude that this procedure can significantly improve both the radiographic appearance and function of the affected knee joint and can also achieve long-term pain relief. They affirm that this procedure may be an alternative treatment option for medial compartment osteoarthritis like HTO or TKR. Baldini et al. [26] presented a study in 2018 in which ten matched pairs of cadaver legs were tested under compression to 1.1 times the body weight comparing an intact knee with proximal fibular osteotomy at $0^{\circ}, 15^{\circ}$ and $30^{\circ}$ of flexion and concluded that the proximal fibular osteotomy decreases the pressure in the medial compartment of the knee, which may reduce knee pain and improve function in patients with medial compartment knee osteoarthritis. In a 2019 retrospective study with radiographic analysis of 560 knees, Wang et al. [27] concluded that FHO produces a reduction in knee adduction moments and rebalances the biceps-proximal fibula-peroneus longus complex reducing pressure on the medial compartment. These biomechanical, radiological and clinical studies support the theory that the direct effect of FHO may be the cause of the longer survival of this group in 
our series, but there are no prospective studies that provide greater clinical evidence for this claim.

This study has several limitations: First, it was a retrospective non-randomized one. However, both groups were similar in terms of demographic, clinical and radiographic variables. Second, the average follow-up is only 109.8 months for FHO and 87.3 months in TFJD group. Therefore, we could not analyze the different effects of both techniques beyond 15 years of follow-up, but the survival analysis seems to show differences in the first 5 years and that these differences are maintained over time.

In conclusion, TFJD associated with CWHTO shows an increase in the conversion to TKR at medium and long-term follow-up with lower HTO survival than CWHTO associated to $\mathrm{FHO}$, especially in patients with a postoperative FTA $\leq 180^{\circ}$ (non-overcorrected). Nevertheless, in the patients not converted to TKR clinical, radiological and satisfaction results are similar for both groups.

Author Contributions: Investigation, A.C., A.F. and B.G.; writing—original draft preparation, J.S.-S.; writing-review and editing, R.T.-C., S.P. and P.H.; supervision, J.C.M. All authors have read and agreed to the published version of the manuscript.

Funding: This research received no external funding.

Institutional Review Board Statement: The study was conducted according to the guidelines of the Declaration of Helsinki, and approved by the Ethics Committee of Parc de Salut Mar Barcelona $(2019 / 8762 / \mathrm{I})$.

Informed Consent Statement: Informed consent was obtained from subjects involved in the study.

Acknowledgments: The authors thank Xavi Duran for assisting with the statistical analysis. This work has been carried out within the framework of the Doctorate of the Department of Surgery and Morphological Sciences, Universitat Autònoma de Barcelona.

Conflicts of Interest: The authors declare no conflict of interest.

\section{References}

1. Loia, M.C.; Vanni, S.; Rosso, F.; Bonasia, D.E.; Bruzzone, M.; Dettoni, F.; Rossi, R. High tibial osteotomy in varus knees: Indications and limits. Joints 2016, 4, 98-110. [CrossRef] [PubMed]

2. McNamara, I.; Birmingham, T.B.; Fowler, P.J; Giffin, J.R. High tibial osteotomy: Evolution of research and clinical applica-tions-A Canadian experience. Knee Surg. Sports Traumatol. Arthrosc. 2013, 21, 23-31. [CrossRef] [PubMed]

3. Filho, R.B.; Xará-Leite, F.; Andrade, R.; Pereira, R.; Schott, V.; Neyret, P.; Espregueira-Mendes, J. Survivorship of high tibial osteotomy: Comparison between opening and closing wedge osteotomy. Ann. Jt. 2018, 3, 52. [CrossRef]

4. Sabzevari, S.; Ebrahimpour, A.; Roudi, M.K.; Kachooei, A.R. High Tibial Osteotomy: A Systematic Review and Current Concept. Arch. Bone Jt. Surg. 2016, 4, 204-212. [PubMed]

5. Brouwer, R.W.; Huizinga, M.R.; Duivenvoorden, T.; Van Raaij, T.M.; Verhagen, A.P.; A Bierma-Zeinstra, S.M.; Verhaar, J.A.N. Osteotomy for treating knee osteoarthritis. Cochrane Database Syst. Rev. 2014. [CrossRef]

6. van Egmond, N.; van Grinsven, S.; van Loon, C.J.M.; Gaasbeek, R.D.; van Kampen, A. Better clinical results after closed- compared to open-wedge high tibial osteotomy in patients with medial knee osteoarthritis and varus leg alignment. Knee Surg. Sports Traumatol. Arthrosc. 2016, 24, 34-41. [CrossRef] [PubMed]

7. Bicer, E.K.; Basa, C.D.; Günay, H.; Aydogdu, S.; Sur, H. The fate of fibular osteotomies performed during high tibial osteotomy. Arch. Orthop. Trauma Surg. 2016, 136, 1085-1090. [CrossRef] [PubMed]

8. Özcan, Ö.; Eroglu, M.; Boya, H.; Kaya, Y. Proximal tibiofibular joint pain versus peroneal nerve dysfunction: Clinical results of closed-wedge high tibial osteotomy performed with proximal tibiofibular joint disruption. Knee Surg. Sports Traumatol. Arthrosc. 2016, 25, 2936-2941. [CrossRef]

9. Torres-Claramunt, R.; Sánchez-Soler, J.F.; Hinarejos, P.; Sala-Pujals, A.; Leal-Blanquet, J.; Monllau, J.C. Proximal Tibiofibular Dis-location in a Closing-Wedge High Tibial Osteotomy Causes Lateral Radiological Gapping of the Knee: A Prospective Ran-domized Study. J. Clin. Med. 2020, 9, 1622. [CrossRef]

10. Sasaki, E.; Akimoto, H.; Iio, K.; Fujita, Y.; Saruga, T.; Kakizaki, H.; Ishibashi, Y. Long-term survival rate of closing wedge high tibial osteotomy with high valgus correction: A 15-year follow-up study. Knee Surg. Sports Traumatol. Arthrosc. 2020, 1-8. [CrossRef]

11. Hui, C.; Salmon, L.J.; Kok, A.; Williams, H.A.; Hockers, N.; van der Tempel, W.M.; Chana, R.; Pinczewski, L.A. Long-term survival of high tibial osteotomy for medial compartment osteoarthritis of the knee. Am. J. Sports Med. 2011, 39, 64-70. [CrossRef] [PubMed] 
12. Polat, G.; Balcı, H.I.; Çakmak, M.F.; Demirel, M.; Şen, C.; Aşık, M. Long-term results and comparison of the three different high tibial osteotomy and fixation techniques in medial compartment arthrosis. J. Orthop. Surg. Res. 2017, 12, 1-7. [CrossRef] [PubMed]

13. Insall, J.N.; Dorr, L.D. Rationale of the Knee Society clinical rating system. Clin. Orthop. 1989, 248, 13. [CrossRef]

14. Ares, O.; Castellet, E.; Maculé, F.; León, V.; Montañez, E.; Freire, A.; Hinarejos, P.; Montserrat, F.; Amillo, J.R. Translation and valida-tion of 'The Knee Society Clinical Rating System' into Spanish. Knee Surg. Sports Traumatol. Arthrosc. 2013, 21, $2618-2624$. [CrossRef]

15. Likert, R. A technique for the measurement of attitudes. Arch. Psychol. 1932, 140, 1-55.

16. Yang, Z.Y.; Chen, W.; Li, C.X.; Wang, J.; Shao, D.C.; Hou, Z.Y.; Gao, S.J.; Wang, F.; Li, J.D.; Hao, J.D.; et al. Medial compartment decompression by fibular osteotomy to treat medial compartment knee osteoarthritis: A Pilot Study. Orthopedics 2015, 38, e1110-e1114. [CrossRef] [PubMed]

17. Zou, G.; Lan, W.; Zeng, Y.; Xie, J.; Chen, S.; Qiu, Y. Early clinical effect of proximal fibular osteotomy on knee osteoarthritis. Bio-Med. Res. 2017, 28, 9291-9294.

18. Wang, X.; Wei, L.; Lv, Z.; Zhao, B.; Duan, Z.; Wu, W.; Zhang, B.; Wei, X. Proximal fibular osteotomy: A new surgery for pain relief and improvement of joint function in patients with knee osteoarthritis. J. Int. Med. Res. 2017, 45, 282-289. [CrossRef]

19. Lu, Z.K.; Huang, C.; Wang, F.; Miao, S.; Zeng, L.; He, S.; Ye, X.; Chen, W. Combination of Proximal Fibulectomy with Arthroscopic Partial Meniscectomy for Medial Compartment Osteoarthritis Accompanied by Medial Meniscal Tear. J. Clin. Diagn. Res. 2018, 12, 1-3. [CrossRef]

20. Liu, B.; Chen, W.; Zhang, Q.; Yan, X.; Zhang, F.; Dong, T.; Yang, G.; Zhang, Y. Proximal fibular osteotomy to treat medial compart-ment knee osteoarthritis: Preoperational factors for short-term prognosis. PLoS ONE 2018, 13, e0197980.

21. Nie, Y.; Ma, J.; Huang, Z.; Xu, B.; Tang, S.; Shen, B.; Kraus, V.B.; Pei, F. Upper partial fibulectomy improves knee biomechanics and function and decreases knee pain of osteoarthritis: A pilot and biomechanical study. J. Biomech. 2018, 71, 22-29. [CrossRef]

22. Qin, D.; Chen, W.; Wang, J.; Lv, H.; Ma, W.; Dong, T.; Zhang, Y. Mechanism and influencing factors of proximal fibular osteotomy for treatment of medial compartment knee osteoarthritis: A prospective study. J. Int. Med. Res. 2018, 46, 3114-3123. [CrossRef] [PubMed]

23. Utomo, D.N.; Mahyudin, F.; Wijaya, A.M.; Widhiyanto, L. Proximal fibula osteotomy as an alternative to TKA and HTO in late-stage varus type of knee osteoarthritis. J. Orthop. 2018, 15, 858-861. [CrossRef] [PubMed]

24. Yazdi, H.; Mallakzadeh, M.; Mohtajeb, M.; Farshidfar, S.S.; Baghery, A.; Givehchian, B. The effect of partial fibulectomy on contact pressure of the knee: A cadaveric study. Eur. J. Orthop. Surg. Traumatol. 2014, 24, 1285-1289. [CrossRef] [PubMed]

25. Huang, W.; Lin, Z.; Zeng, X.; Ma, L.; Chen, L.; Xia, H.; Zhang, Y. Kinematic characteristics of an osteotomy of the proximal aspect of the fibula during walking: A case report. JBJS Case Connect. 2017, 7, e43. [CrossRef]

26. Baldini, T.; Roberts, J.; Hao, J.; Hunt, K.; Dayton, M.; Hogan, C. Medial compartment decompression by proximal fibular osteot-omy: A biomechanical cadaver study. Orthopedics 2018, 41, e496-e501. [CrossRef]

27. Wang, J.; Lv, H.; Chen, W.; Fan, M.; Li, M.; Zhang, Y. Anatomical Adaptation of Fibula and its Mechanism of Proximal Partial Fibulectomy Associated with Medial Compartment Knee Osteoarthritis. Orthop. Surg. 2019, 11, 204-211. [CrossRef] 